\title{
A Diagram Depicting Forecast Skill and Predictability
}

\author{
Richard A. Anthes and David P. Baumhefner
}

National Center for Atmospheric Research, ${ }^{1}$ Boulder, CO 80307

\begin{abstract}
In operational numerical weather prediction systems, both observations and numerical models contribute to the skill of the forecast. A simple diagram representing the relative contributions of observations and models to the current level of forecast skill and to the ultimate predictability of atmospheric phenomena is interpreted in this note. The forecast skill of $500 \mathrm{mb}$ heights and an estimate of the ultimate predictability of this variable are used in a quantitative illustration of the diagram.
\end{abstract}

\section{Introduction}

Numerical weather prediction models, together with improved observational and analysis systems, have led to steady improvements in operational forecasts of synopticscale weather circulations over the past 25 years (Shuman, 1978). It is well known, however, that there is an inherent limit to the predictability of atmospheric motions because of the growth of initial errors in unresolvable (or unobservable) scales of motion. The growth of these errors eventually will contaminate all scales of motion, even with a "perfect" numerical model (a model that contributes no errors to a forecast).

Recent advances in scientific understanding and in technology (remote-sensing systems from the ground and space and supercomputers) have made it possible to envisage significant improvements in predicting mesoscale atmospheric events (UCAR, 1983). This possibility raises the important questions of what the inherent limits to mesoscale predictability are, and what practical barriers in the current observational system and numerical models must be removed in order to approach these limits in a future operational system. To help answer these questions, it is useful to separate the relative contributions of observations and models to forecast skill.

\footnotetext{
${ }^{1}$ Manuscripts for focus on forecasting should be sent directly to Dr. Robert Burbee, editor, focus on forecasting, Hurricane Research Division, AOML, 4301 Rickenbacker Causeway, Miami, FL 33149.

${ }^{2}$ The National Center for Atmospheric Research is sponsored by the National Science Foundation.
}

두 1984 American Meteorological Society

\section{Quantitative Contribution of Observations and Models to Forecast Skill}

A recent document describing the scientific and technological bases for a national program in mesoscale meteorology (UCAR, 1983) included a schematic diagram illustrating the relative contribution of observations and models to the description or predictability of atmospheric phenomena. This figure, reproduced in Fig. 1, and the accompanying discussion in the UCAR document were incomplete and somewhat vague in that no specific examples were given to illustrate quantitatively how such a diagram could be computed. As a result, there has been some confusion in the interpretation of Fig. 1. The purpose of this note is to illustrate, for a particular aspect of atmospheric structure (the synoptic-scale 500 mb height field), how a quantitative diagram corresponding to the schematic shown in Fig. 1 can be constructed, and to illustrate the relative contribution of observations and mod-

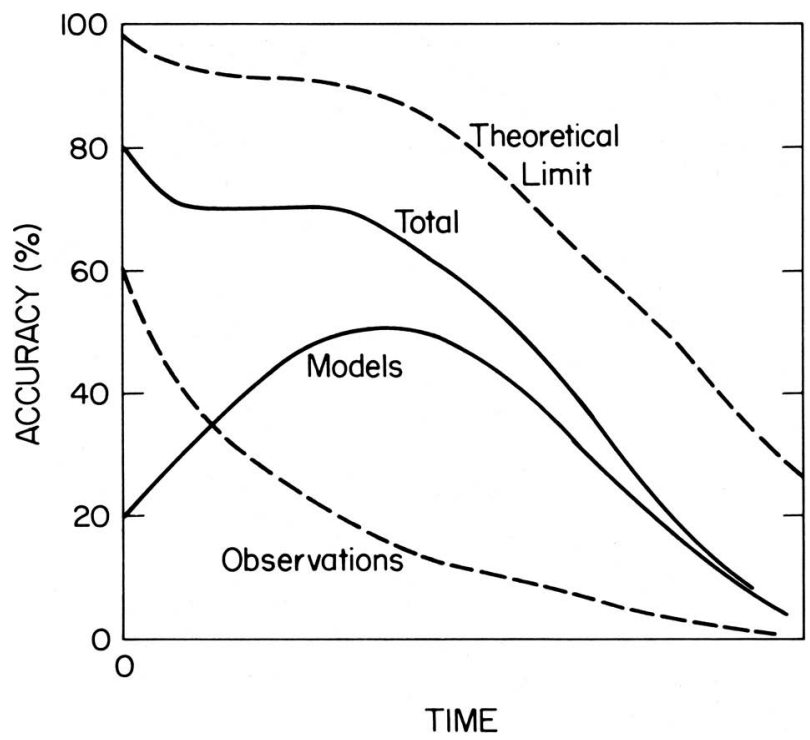

FIG. 1. Schematic diagram illustrating relative contributions with increasing time of observations and models to resolving the structure of an arbitrary atmospheric phenomenon. On the diagram, $100 \%$ represents a perfect knowledge of the circulation system (UCAR, 1983). 


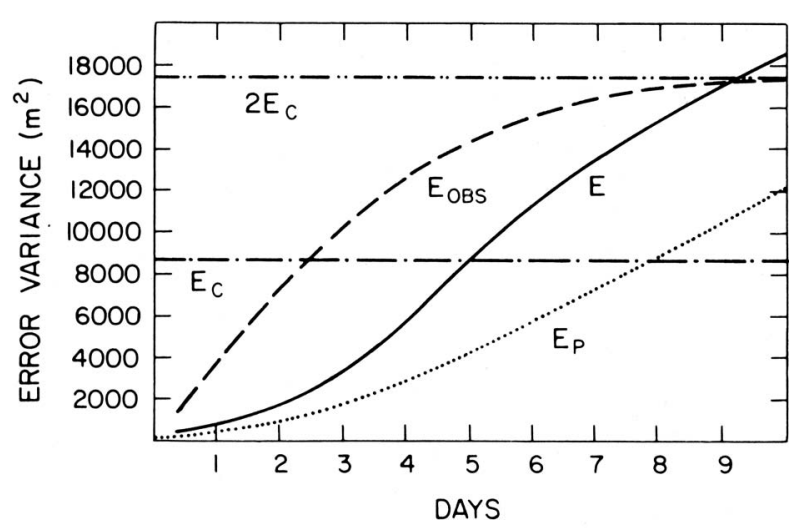

FIG. 2. Growth of error variance of $500 \mathrm{mb}$ heights. $E_{c}$ represents the climatological error variance; $E_{\mathrm{obs}}$, a persistence forecast; $E$, a forecast with a model of skill approximately equal to that of present operational models; and $E_{p}$, the predictability error growth (which represents an estimate of the maximum obtainable forecast skill).

els to forecast skill and predictability of this feature.

Studies of the predictability of large-scale atmospheric motions typically have compared the growth rate of model errors (for example, the error variance of $500 \mathrm{mb}$ heights) with the error variance associated with a persistence forecast and with the climatological error variance. An example is shown in Fig. 2, which represents an ensemble average of 10 forecasts from the National Center for Atmospheric Research's (NCAR) global community forecast model and predictability estimates from the community climate model (Williamson, 1983). In Fig. 2, $E_{\text {obs }}$ represents the error variance of $500 \mathrm{mb}$ heights associated with a persistence forecast based on an analysis of observations at time $t_{0}$. In operational centers, this analysis may be aided by a numerical model that contributes information either by providing a first-guess field from a previous forecast or by assimilating observations before time $t_{0}$. The curve labeled " $E$ " represents the error variance associated with a model forecast initialized with the analysis at $\mathrm{t}_{0}$. The lowest curve, $E_{p}$, illustrates an estimate of the predictability error growth. This curve represents the growth of small differences in two forecasts using identical models and is assumed to represent the growth of forecast errors in a perfect model. Baumhefner (1984) describes in more detail how the predictability estimate, $E_{p}$, is determined. The climatological variance, $E_{c}$, also is plotted, as well as the asymptotic limit of skill, $2 E_{c}$ (Thompson, 1961). Note that $E$ does not approach $2 E_{c}$ asymptotically (as does $E_{p}$ ), but to a slightly higher value. The forecast model error contains an additional contribution to the error variance because of the difference between the model's climatology and the observed climatology. $E_{c}$ is a meaningful limit to forecast skill for large-scale flows and large ensemble averages.

Using the variables found in Fig. 2, we may write an equation for the reduction of the forecast error variance below the climatological error variance, $E_{c}$, due to the analysis of the observations at $t_{0}$ and the additional reduction due to the model system,

$$
E(t)=E_{c}-\Delta E_{\mathrm{obs}}-\Delta E_{\mathrm{mod}},
$$

where

$$
\begin{aligned}
\Delta E_{\mathrm{obs}} \equiv & \text { reduction of error variance below } E_{c} \text { at any time } \\
& \text { due to the analysis of observations at time } t_{0} . \text { The } \\
& \text { quantity } E_{c}-\Delta E_{\mathrm{obs}} \text { represents a persistence } \\
& \text { forecast. } \\
\Delta E_{\mathrm{mod}} \equiv & \text { reduction of } E_{\mathrm{obs}}(t) \text { at any time due to the model; }
\end{aligned}
$$

$$
\begin{aligned}
\Delta E_{\mathrm{obs}} & =E_{c}-E_{\mathrm{obs}}(t) ; \\
\Delta E_{\mathrm{mod}} & =E_{\mathrm{obs}}(t)-E(t) .
\end{aligned}
$$

From Eq. (1) we may write an equation defining forecast skill, $F$, as

$$
F(t) \equiv 1-\frac{E(t)}{E_{c}}=\frac{\Delta E_{\mathrm{obs}}}{E_{c}}+\frac{\Delta E_{\mathrm{mod}}}{E_{c}} .
$$

Equation (4) illustrates clearly the relative contributions of observations and models to forecast skill. A quantity similar to $F$ as defined by Eq. (4) is the theoretical predictability, $P$, which may be considered as the upper bound to forecast skill. Predictability may be defined as

$$
P(t)=1-\frac{E_{p}(t)}{E_{c}},
$$

where $E_{p}(t)$ is the estimated lower bound to the error variance as a function of time, as estimated by the growth of small differences in initial conditions for a numerical model (Fig. 2).

Figure 3 shows a quantitative diagram illustrating forecast skill, predictability, and the contributions of observations and models to forecast skill, based on the data used to construct Fig. 2. A quantitative analog to the curves shown in Fig. 3 is given in Table 1. In Table 1 and Fig. 3, the curve $\Delta E_{\text {pmod }} / E_{c}$ represents the contribution to a theoretical limit to forecast skill by a perfect model.

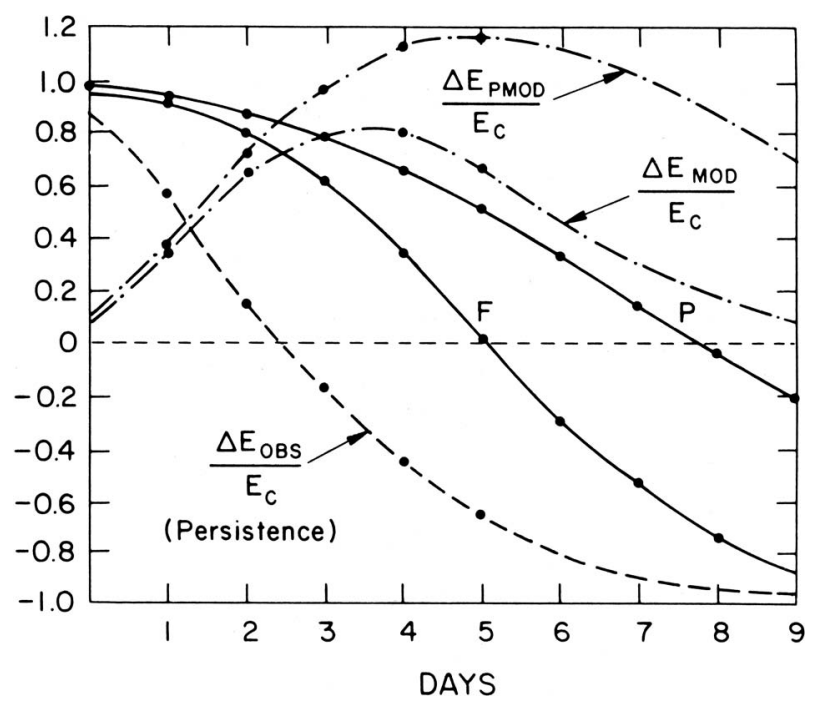

FIG. 3. Quantitative diagram corresponding to Fig. 1 for forecast skill and predictability of $500 \mathrm{mb}$ heights based on the same data used to compute the curves in Fig. 2(Table 1). The curves are defined and interpreted in the text. 


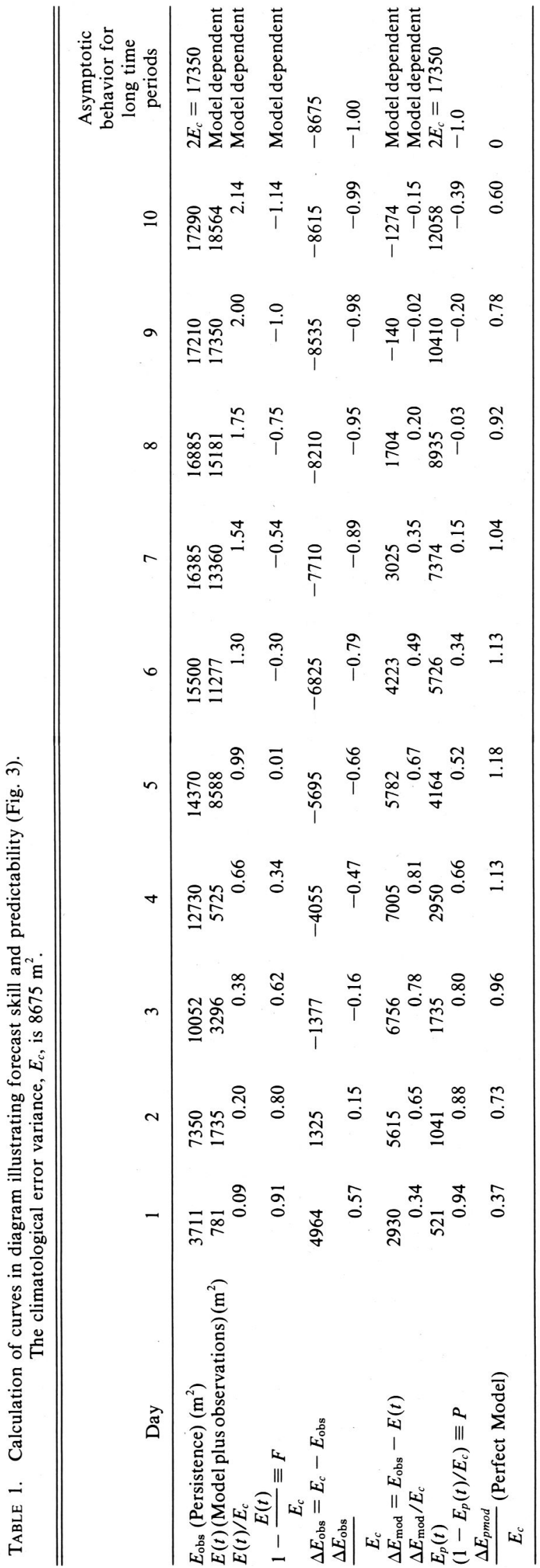

\section{Discussion}

The relative role of observations and numerical models in the prediction of the $500 \mathrm{mb}$ heights can be visualized by considering Fig. 3. At the initial time $(t=0)$, observations provide most of the information. For the early part of the forecast period, the contribution of the observations exceeds that of the model in providing information on the $500 \mathrm{mb}$ heights; however, this contribution decays rapidly during the first few days, while the model's contribution to forecast skill and predictability increases. Eventually, all skill and predictability are lost because of inherent uncertainties in the initial conditions.

The information contained in Fig. 3 is, of course, essentially the same as that presented in Fig. 2. The difference is that the forecast skill and predictability depicted in Fig. 3 are normalized between \pm 1.0 . (A choice of $2 E_{c}$ rather than $E_{c}$ in Eqs. (1)-(4) would have led to a normalization between 0 and 1.0 , as depicted in Fig. 1.) Similar figures with the same scaling could be constructed for other variables, such as temperature, winds, or moisture content. It is likely that forecast skill and the limits to predictability of these and other variables would differ from that of the $500 \mathrm{mb}$ height field.

Acknowledgments. Akira Kasahara and Philip Thompson contributed helpful suggestions on the manuscript. Ann Modahl provided capable typing and editorial assistance.

\section{References}

Baumhefner, D. P., 1984: The relationship between present largescale forecast skill and new estimates of predictability error growth. In Predictability of Fluid Motions, edited by Greg Holloway and Bruce J. West, American Institute of Physics, New York, pp. $169-180$.

Shuman, F. G., 1978: Numerical weather prediction. Bull. Amer. Meteor. Soc., 59, 5-17.

Thompson, P. D., 1971: Numerical Weather Analysis and Prediction. Macmillan Co., New York, 170 pp.

UCAR, 1983: The National STORM program-Scientific and technological bases and major objectives. Report submitted by the University Corporation for Atmospheric Research, Boulder, Colo., to the National Oceanic and Atmospheric Administration in fulfillment of Contract NA81RACOO123. Edited by R. A. Anthes, National Center for Atmospheric Research (which is sponsored by the National Science Foundation), Boulder, Colo., $520 \mathrm{pp}$.

Williamson, D. L., 1983: Description of the NCAR Community Climate Model (CCMOB). NCAR Tech. Rept. NCAR/TN-0+STR, $88 \mathrm{pp}$. (Available from the National Center for Atmospheric Research, Boulder, CO 80307.) 